\title{
Two leg quantum Ising ladder: A bosonization study of the ANNNI model
}

\author{
D. Allen $\dagger$, P. Azaria $\dagger$, and P. Lecheminant $\ddagger$ \\ $\dagger$ Laboratoire de Physique Théorique des Liquides, Université Pierre et Marie \\ Curie, 4 Place Jussieu, 75252 Paris, France \\ ¥ Laboratoire de Physique Théorique et Modélisation, Université de \\ Cergy-Pontoise, 5 Mail Gay-Lussac, Neuville sur Oise, 95301 Cergy-Pontoise \\ Cedex, France
}

\begin{abstract}
.
The quantum ANNNI chain in a transverse field is investigated by means of the bosonization approach in the limit of large next-nearest neighbor interaction. In this regime, this model can be viewed as a weakly coupled two-leg zigzag ladder which enables us to derive its low energy effective field theory. In particular, it is shown that the effect of frustration in the system is captured by the presence of a non zero conformal spin perturbation that accounts for the existence of all the incommensurate phases of the model.
\end{abstract}

PACS numbers: 75.10.Jm, 64.70.Rh 
One of the most striking effect of frustration in magnetic systems is that it can lead to a huge number of degenerate ground states. As a result, the nature of finite temperature phases may not, in contrast with ferromagnetic systems, be linked only to the sole nature of the microscopic degrees of freedom and the dimension of space-time. Since frustration induces strong fluctuations that involve large number of spins, the low energy physics is determined by interferences between very different regions of phase space. With this picture in mind one may not expect the field theoretical description of the frustration to be an easy task. Of course there have been several attempts to describe frustrated magnets by field theories. But, to our knowledge they were mostly restricted to models that displayed well defined ground states, such as helical ordering, and the main effect of frustration was captured by an enlargement of the dimension of the order parameter space 1]. Here our aim is to single out an operator, in the Renormalization Group (RG) sense, that captures the effect of frustration. In this respect, we shall consider the most studied frustrated system i.e. the two dimensional ANNNI model (see Refs. [2, 3, - 1 for a review). This model is characterized by a nearest-neighbor ferromagnetic interaction $\left(-J_{1}<0\right)$ and a competiting next-nearest neighbor antiferromagnetic interaction $\left(J_{2}>0\right)$ :

$$
\mathcal{H}=-J_{1} \sum_{(i, j)} \sigma_{i} \sigma_{j}+J_{2} \sum_{[k, l]} \sigma_{k} \sigma_{l},
$$

where $(i, j)$ denotes nearest neighbor pairs and $[k, l]$ next-nearest ones along a single space direction. The phase diagram of the model (1) is very rich and well known and can be summarize as follows. At zero temperature, the ground state is ferromagnetic for $J_{2}<J_{1} / 2$ and antiferromagnetic (actually an antiphase $\uparrow \uparrow \downarrow \downarrow$ ) for $J_{2}>J_{1} / 2$. At the special point $J_{2}=J_{1} / 2$, the ground state is infinitely degenerate and correlation functions are short ranged. At finite temperatures $(T \neq 0)$, one observes five different phases depending on the parameters (see figure (1)): ferromagnetic $(\mathrm{F})$, paramagnetic commensurate $(\mathrm{PC})$, paramagnetic incommensurate $(\mathrm{PI})$, incommensurate critical phase (IC, also usually called "floating phase") and antiphase (A). Three different kind of phase transitions are present in this diagram: An Ising transition between the $\mathrm{F}$ and $\mathrm{PC}$ phases, a commensurate-incommensurate transition[5] between the $\mathrm{A}$ and IC phases, and a Berezinski-Kosterlitz-Thouless (BKT) transition separates the IC and PI phases. There is also a disorder line that extends down to zero temperature which divides the PC and PI phases [6]. As a most notable feature of this phase diagram is the existence of an incommensurate critical phase in a finite region of the parameter space. In a seminal work, Villain and Bak [7] have proposed an approximate effective theory in terms of fermions valid in the vicinity of the degenerate point $J_{2}=J_{1} / 2$. All their predictions have been further confirmed by numerical investigations and series expansions. It is the purpose of the present letter to propose a complementary and alternative low energy description of the ANNNI model valid in the large $J_{2}$ limit. Furthermore, within our approach, we exhibit a particular operator that is at the origin of the incommensurate phases found in the phase diagram (figure (1)).

Our starting point is to map (11) into a quantum Ising model in a transverse magnetic field. This is done by introducing an anisotropy between the nearest neighbor interaction in the $x$-direction $\left(J_{1}\right)$ and in the $y$-direction $\left(J_{\tau}\right)$. As it is well known (see Ref. [4] for a review), the physics of the classical model (1) should be equivalent to the one described by the following quantum Hamiltonian:

$$
\mathcal{H}=\sum_{n}\left[-\beta J_{\tau}^{*} \sigma_{n}^{z}+\beta J_{2} \sigma_{n}^{x} \sigma_{n+2}^{x}-\beta J_{1} \sigma_{n}^{x} \sigma_{n+1}^{x}\right],
$$




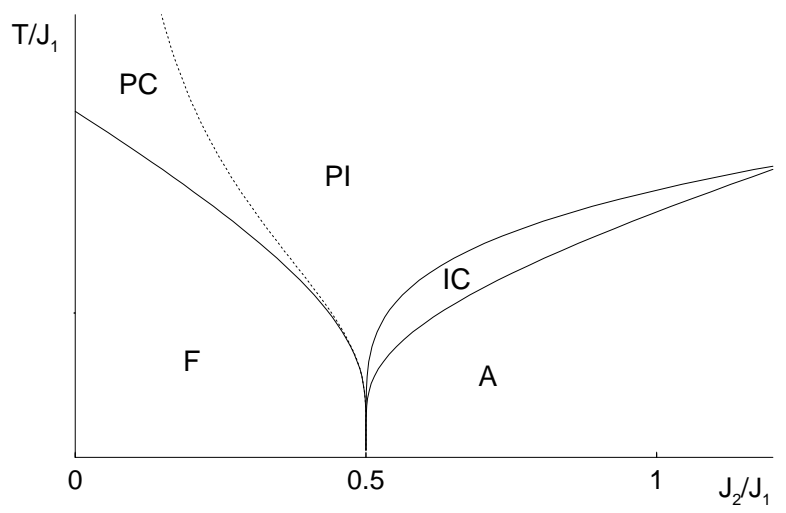

Figure 1. Phase diagram of the classical two-dimensional ANNNI model.

where $\sigma_{n}^{x}, \sigma_{n}^{z}$ are Pauli matrices and $2 \beta J_{\tau}^{*}=\ln \operatorname{coth}\left(\beta J_{\tau}\right)$. The model (2) is nothing but the one-dimensional axial next-nearest neighbor Ising (ANNNI) model in a transverse field. As seen in figure (2), it can also be viewed as two quantum Ising chains labelled (1) and (2) coupled by a "zigzag" interaction with strength $J_{1}$ and thus identifies with a two-leg quantum Ising ladder with Hamiltonian:

$$
\begin{aligned}
\mathcal{H}= & \sum_{n, a=1,2}\left[-\beta J_{\tau}^{*} \sigma_{a}^{z}\left(n+\frac{a}{2}\right)-\beta J_{2} \sigma_{a}^{x}\left(n+\frac{a}{2}\right) \sigma_{a}^{x}\left(n+1+\frac{a}{2}\right)\right] \\
& -\frac{\beta J_{1}}{2} \sum_{n} \sigma_{1}^{x}\left(n+\frac{1}{2}\right)\left[\sigma_{2}^{x}(n)-\sigma_{2}^{x}(n+1)\right]+(1 \rightarrow 2) .
\end{aligned}
$$

Notice that in order to obtain (3) we have performed an (unphysical) gauge transformation on the ath chain $(a=1,2): \sigma_{a}^{x}(n+a / 2) \rightarrow(-1)^{n+a} \sigma_{a}^{x}(n+a / 2)$. As we shall now see, the model (3) can be consistently described by a continuous field theory in the limit $J_{1}<<J_{2}$ and $J_{2} \sim J_{\tau}^{*}$.

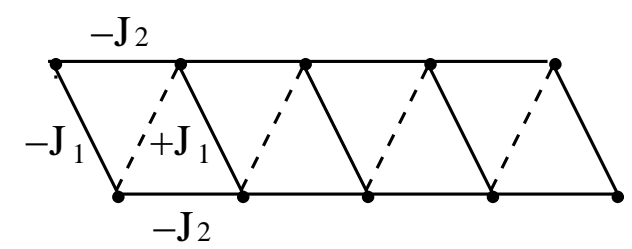

Figure 2. In the large $J_{2}$ limit, the ANNNI model is better seen as two weakly coupled quantum Ising chains.

Continuum limit. We shall study the model in the vicinity of the antiferromagnetic phase where $J_{1}<<J_{2}$. One can take advantage that in the limit $J_{1}=0$ the model is equivalent to two decoupled Ising models which are critical when $J_{2}=J_{\tau}^{*}$. One can therefore expand the theory around the conformal invariant fixed point with symmetry $\mathrm{Z}_{2} \otimes \mathrm{Z}_{2}$. In the critical regime the two Ising chains are described 
by two pairs of right and left Majorana (real) fermions $\psi_{a(R, L)}, a=1,2$ :

$$
\mathcal{H}_{0}=-i \frac{v}{2} \sum_{a=1,2}\left(\psi_{a R} \partial_{x} \psi_{a R}-\psi_{a L} \partial_{x} \psi_{a L}\right)-m\left(\epsilon_{1}+\epsilon_{2}\right),
$$

$\epsilon_{a}=i \psi_{a R} \psi_{a L}$ being the energy operator of the ath Ising model whereas the mass gap and the velocity are given by: $m=2\left(J_{\tau}^{*}-J_{2}\right) / T<<1, v=a_{0} J_{\tau}^{*} / T$ ( $a_{0}$ being the lattice spacing). In the absence of interchain interaction, the system is disordered (respectively ordered) when $m>0$ (respectively $m<0$ ) and one has $\left\langle\sigma_{a}\right\rangle=0$ (respectively $\left\langle\sigma_{a}\right\rangle \neq 0$ ) where $\sigma_{a}$ are the order operators associated with the two Ising models. We consider now the interacting case and take the continum limit of the second term in equation (3) in the regime $J_{1} \ll J_{2}$ to obtain:

$$
\mathcal{H} \simeq \mathcal{H}_{0}+g \mathcal{V}, \quad \mathcal{V}=a_{0}^{1 / 4}\left(\sigma_{1} \partial_{x} \sigma_{2}-\sigma_{2} \partial_{x} \sigma_{1}\right) .
$$

When $m=0$ and $g=J_{1} / 2 T=0$, the model is conformal invariant with central charge $c=\frac{1}{2}+\frac{1}{2}=1$. In the generic case, this fixed point is perturbed by the thermal operators $\epsilon_{1(2)}$ which have scaling dimension $\Delta=1$ and the operator $\mathcal{V}$ which has scaling dimension $\Delta=5 / 4$. It is important to stress that both operators reflect very different physical behaviors. While the former is the standard operator measuring deviation from criticality in non-frustrated Ising magnets, the latter encodes the whole effect of frustration and is responsible of the non trivial phases of the ANNNI model as we shall see. The operator $\mathcal{V}$ manifests itself by the fact that it is a parity symmetry breaking perturbation with a conformal spin equals to 1 . The effect of such non-zero conformal spin term is non trivial since the usual irrelevant versus relevant criterion does not hold for such a non-scalar contribution (see for instance the discussion in Ref. [8]). In fact, a similar operator also appears in the study of the $S=1 / 2$ Heisenberg zigzag ladder 9] and has been called a "twist term". Such contribution is difficult to handle non-perturbatively but it has been stressed that this term may represent a new mechanism for incommensuration in one-dimensional systems [9, 10]. As we shall see, in the particular case of the ANNNI model, the effect of this operator can be elucidated by means of the bosonization approach and accounts for the formation of the non trivial incommensurate phases depicted in figure (1).

Bosonization and Effective Theory. We shall use the well known equivalence between two critical Ising models, characterized by four chiral real fermions $\psi_{1 R(L)}$ and $\psi_{2 R(L)}$, and a free boson theory described by the chiral fields $\phi_{R(L)}$. The bosonization rules are (see Ref. [8] for a review):

$$
\left(\psi_{1}+i \psi_{2}\right)_{R(L)}=\frac{1}{\sqrt{\pi a_{0}}} \exp \left( \pm i \sqrt{4 \pi} \phi_{R(L)}\right)
$$

from which it follows that $\mathcal{H}_{0}$ is equivalent to a sine-Gordon model at $\beta^{2}=4 \pi$ (free fermion point). The bosonization of the twist term (5) requires more work. A suitable bosonic expression is obtained by considering the following operator product expansion (OPE) that stems from the fact that the order operator $\sigma_{a}$ is a primary field:

$$
\left(T_{1}-T_{2}\right)(z) \sigma_{1} \sigma_{2}(w, \bar{w}) \sim \frac{1}{z-w}\left[\sigma_{2} \partial \sigma_{1}-\sigma_{1} \partial \sigma_{2}\right](w, \bar{w}),
$$

where $T_{a}(a=1,2)$ are the energy momentum tensors in the holomorphic sector $(z=v \tau+i x)$ associated with the Ising models. The fields in the left hand side of equation (7) can be expressed in terms of the bosonic fields: $T_{1}-T_{2}=\cos \left(\sqrt{16 \pi} \phi_{L}\right)$ 
and $\sigma_{1} \sigma_{2}=\sqrt{2} \sin (\sqrt{\pi} \Phi)\left(\Phi=\phi_{L}+\phi_{R}\right)$. We thus deduce by performing the OPE in the bosonic theory the following representation of the twist term:

$$
\mathcal{H}=\frac{v}{2}\left(\left(\partial_{x} \Phi\right)^{2}+\left(\partial_{x} \Theta\right)^{2}\right)-\frac{m}{\pi a_{0}} \cos (\sqrt{4 \pi} \Phi)-\mathrm{i} \frac{g \sqrt{2}}{a_{0}} \cos (\sqrt{\pi} \Phi) \sin (\sqrt{4 \pi} \Theta),
$$

$\Theta=\phi_{L}-\phi_{R}$ being the dual field. The next step of our approach is to map (8) onto the XXZ Heisenberg model in a magnetic field:

$$
\mathcal{H}=\frac{2 \pi u}{3}\left(\mathbf{J}_{R}^{2}+\mathbf{J}_{L}^{2}\right)+4 \pi m a_{0}\left(J_{R}^{x} J_{L}^{x}+J_{R}^{y} J_{L}^{y}\right)+\pi g \sqrt{2}\left(J_{R}^{y}-J_{L}^{y}\right)+2 \pi \lambda_{0} J_{R}^{z} J_{L}^{z},
$$

where $u=5 v / 4, \lambda_{0}=3 v / 2$ and the $\mathrm{SU}(2)_{1}$ Kac-Moody currents are given by $J_{L, R}^{z}=\partial_{x} \phi_{L, R} / \sqrt{2 \pi}$ and $J_{L, R}^{+}=\exp \left( \pm i \sqrt{8 \pi} \phi_{L, R}\right) /\left(2 \pi a_{0}\right)$. The two expressions (8) and (9) can be shown to be equivalent by a canonical transformation at the special value $\lambda_{0}=3 v / 2$. As a result, in an appropriate basis, the twist operator acts as a magnetic field. The model (9) is more conveniently analyzed in a basis where the magnetic field lies along the $z$ axis. To do so, we perform a $\pi / 2$ rotation around the $\mathrm{x}$-axis in the spin space so that one finally obtains after rebosonizing once again the currents:

$$
\mathcal{H}=\frac{u^{*}}{2}\left(\left(\partial_{x} \Phi\right)^{2}+\left(\partial_{x} \Theta\right)^{2}\right)-g_{1} \cos (\sqrt{8 \pi Q} \Phi)-g_{2} \cos (\sqrt{8 \pi / Q} \Theta)-h \partial_{x} \Theta
$$

where

$$
Q=\sqrt{\frac{1-m a_{0} / u}{1+m a_{0} / u}}, \quad u^{*}=u \sqrt{\left(1+m a_{0} / u\right)\left(1-m a_{0} / u\right)},
$$

$g_{1(2)}=\left(2 m \pm \lambda_{0} / a_{0}\right) /\left(4 \pi a_{0}\right)$, and $h=g \sqrt{\pi / Q}$. The effective field theory (10) is the main result of this work and all the different phases (apart from the $\mathrm{F}$ and $\mathrm{PC}$ phases) observed in the ANNNI model can be deduced from a simple analysis of it.

Phase Diagram. Consider first the high-temperature phase, i.e. when $m>0$. Since $Q<1$, for sufficiently large $m$, the term $\cos (\sqrt{8 \pi / Q} \Theta)$ is strongly irrelevant and can be dropped. The remaining theory is then easy to analyse and a mass gap to all excitations is generated due to the presence of the relevant $\cos (\sqrt{8 \pi Q} \Phi)$ term. On the other hand the operator $\partial_{x} \Theta$ leads to incommensurate fluctuations of the $\Theta$ dependent correlation functions. This phase corresponds to the incommensurate paramagnetic phase PI. This picture is confirmed by an exact solution at the special value $Q=1 / 2$ where the model becomes equivalent to that of free fermions with dispersion $\epsilon_{ \pm}^{2}(k)=v^{2}(k \pm g \sqrt{2} \pi / v)^{2}+9 v^{2} / 16 a_{0}^{2}$. As readily seen, there is a spectral gap and incommensuration develops as soon as $g \neq 0$ with wavevector $k^{*}=\sqrt{2} \pi g / v$.

Similarily one can study the low temperature regime i.e. $m<0$ and $Q>1$. For sufficiently large $m$, it is now the $\cos (\sqrt{8 \pi Q} \Phi)$ term that is irrelevant and can be dropped. After a duality transformation, the resulting Hamiltonian is equivalent to the XXZ chain in a magnetic field along the $z$ axis which is equivalent to the bosonized version of the Villain-Bak theory 11 derived in the vicinity of the degenerate point $J_{2} / J_{1}=1 / 2, T=0$. At small $g$, there is a gap to all excitations with no incommensuration: it corresponds to the A phase. As $g$ grows, the magnetic field increases until it reaches the gap at some critical value $\left(g_{c 1}\right)$ above which the excitations become massless. For $g>g_{c 1}$, the system displays as well an incommensurate behavior with wavevector $k^{*} \sim \sqrt{g^{2}-g_{c 1}^{2}}$ and one enters the floating phase IC. The nature of the transition at $g=g_{c 1}$ is of a commensurate-incommensurate type [5. Finally, as $g$ further increases, the $\cos (\sqrt{8 \pi Q} \Phi)$ term eventually becomes 
relevant and opens a gap at a critical value $g_{c 2}$ where a BKT transition to the PI phase takes place. This picture is, as above, confirmed by an exact solution at the point $Q=2$ where the model (10) becomes equivalent to massive free fermions.

As seen, the previous analysis correctly reproduces the phase diagram of the ANNNI model in the vicinity of the A phase where frustration plays its tricks. To do so, we have assumed that $|m|$ was sufficiently large to be able to neglect one of the cosine terms in equation (10). One can question the validity of this scheme when $m \sim 0$ where both $\cos (\sqrt{8 \pi Q} \Phi)$ and $\cos (\sqrt{8 \pi / Q} \Theta)$ operators, being almost marginal, compete. A detailed analysis of the RG equations associated with (10) is thus called for but one is faced with the difficulty that the coupling constants are not small since $\lambda_{0}$ is of order one. One has therefore to make the hypothesis that the qualitative feature of the RG approach does not depend on the strength of $g_{1}$ and $g_{2}$ and treat them as small couplings. The RG equations have already been obtained in a different context by Giamarchi and Schulz [12]. It follows from [12] that the results we have obtained for large $|m|$ remain valid for small $m$ confirming our hypothesis. However, there is room for a spin-flop transition from the A phase directly to the PI phase if the $\cos (\sqrt{8 \pi Q} \Phi)$ perturbation blows up before the $\partial_{x} \Theta$ term closes the gap. The occurence of such a transition strongly depends on the microscopic couplings of the bare Hamiltonian. The analysis of the RG equations for $\lambda_{0}=6 u / 5$ reveals that a spin flop transition does not occur for the ANNNI model. However, care has to be payed since for this value of $\lambda_{0}$, perturbation theory strictly does not apply and this leaves open the question of the existence of a Lifshitz point in the ANNNI model. Furthermore, notice that the presence of a Lifshitz point is a generic feature of (10). Therefore one may expect that other lattice Hamiltonians displaying the same qualitative phase diagram than the ANNNI model will exhibit such a point. In summary, we have derived a low energy description of the ANNNI model in the large $J_{2}$ limit where the system can be viewed as a weakly coupled two-leg zigzag ladder. This enables us to start from a conformal invariant fixed point in the vicinity of which the continuum limit is well defined and frustration manifests itself through the twist operator. This approach accounts for all the incommensurate phases of the ANNNI model and in the low temperature limit matches the Villain-Bak description near the degenerate point.

D. Allen is a CNRS post-doctoral fellow and thanks l'université Cergy-Pontoise for hospitality while this work was initiated.

[1] Magnetic Systems with Competiting Interactions, edited by H. T. Diep, World Scientific (1994).

[2] W. Selke, Phys. Rep. 170, 213 (1988); W. Selke in Phase Transition and Critical Phenomena ed. C. Domb and J. L. Lebowitz, Vol. 15, Academic Press, New York (1992).

[3] J. Yeomans, in Solid State Physics, Vol. 41, Academic Press, New York (1987), Eds. H. Ehrenreich and J. L. Turnbull.

[4] B. K. Chakrabarti, A. Dutta, and P. Sen, Quantum Ising Phases and Transitions in Transverse Ising Models, Springer-Verlag, 1996.

[5] G. I. Dzhaparidze and A. A. Nersesyan, JETP Lett. 27, 334 (1978); V. L. Pokrovsky and A. L. Talapov, Phys. Rev. Lett. 42, 65 (1979).

[6] I. Peschel and V. J. Emery, Z. Phys. B 43, 241 (1981).

[7] J. Villain and P. Bak, J. Physique 42, 657 (1981).

[8] A. O. Gogolin, A. A. Nersesyan, and A.M. Tsvelik, The bosonization approach to strongly correlated systems (Cambridge University Press, Cambridge, 1998).

[9] A. A. Nersesyan. A. O. Gogolin. and F. H. L. Essler, Phys. Rev. Lett. 81, 910 (1998).

[10] A. M. Tsvelik, cond-mat/0011268.

[11] F.D.M. Haldane, P. Bak, and T. Bohr, Phys. Rev. B 28, 2743 (1983).

[12] T. Giamarchi and H. J. Schulz, J. Phys. France 49, 819 (1988). 